\title{
Ion acceleration in supersonically rotating magnetized-electron plasma
}

\author{
N J Fisch ${ }^{1}$, Y Raitses ${ }^{1}$ and A Fruchtman ${ }^{2}$ \\ ${ }^{1}$ Princeton Plasma Physics Laboratory, Princeton University, Princeton, NJ, USA \\ ${ }^{2}$ H. I. T. - Holon Institute of Technology, Holon, Israel \\ E-mail: fisch@princeton.edu
}

Received 6 September 2011, in final form 10 October 2011

Published 14 November 2011

Online at stacks.iop.org/PPCF/53/124038

\begin{abstract}
A leading method of propelling plasma is through the electrical acceleration of ions through a cloud of rotating electrons, where the rotating electrons are held in place axially by a magnetic filter. However, in certain parameter regimes, devices based on this propulsion principle appear to work far better than they should, at least based on the accepted design principles. This unexpected fortunate performance is explained here by self-organizing features of supersonically rotating electron plasma. In fact, several ion acceleration mechanisms that narrow the plume can be identified. These useful acceleration mechanisms, which persist even as the electron temperature vanishes, are newly identified here and are common to rotating electron plasmas in a variety of settings.
\end{abstract}

(Some figures may appear in colour only in the online journal)

\section{Introduction}

A wide variety of plasma devices employ rotating plasma. In the case of radiation devices, such as magnetrons, the plasmas are comprised of only one species component, electrons. Rotating in crossed electric and magnetic fields, these electrons fall through an electric potential, thus releasing energy to the waves enabling the crossing of the confining magnetic field lines. For Hall-effect thrusters, the plasma is overall neutral, with approximately equal number of ions and electrons, with only the electrons rotating. In the case of centrifugal mirror confinement, or in the case of plasma centrifuges, both the electrons and ions rotate, in fact, co-rotate, with approximately the same angular frequency.

These devices operate in very different regimes of plasma physics, both with respect to density and temperature, and clearly with respect to magnetization of the different plasma components. Certainly the physical apparatus and applications in these regimes are very different as well. Yet they all share interesting effects by virtue of their similar geometry and 


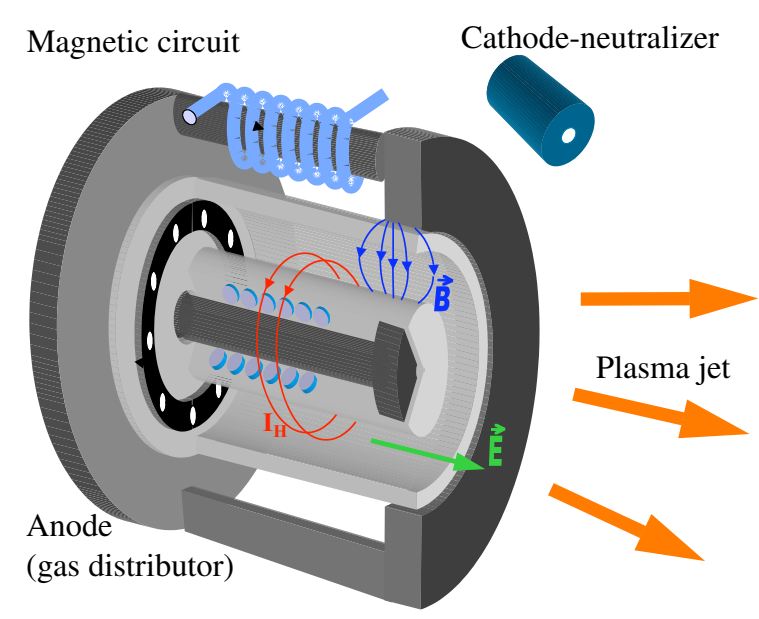

Figure 1. Conventional Hall thruster.

by virtue of the rotation. In each case, the magnetic field lines form magnetic surfaces with symmetry in the azimuthal direction, which is the direction of the rotation of the magnetized electrons. In each case, the magnetized electrons are largely collisionless on the time scales of interest. It is generally assumed that in regimes of interest the magnetized electrons tend to equilibrate along the magnetic surfaces, so that the magnetic surfaces also tend to be equipotential surfaces (see, for example, [1]).

Indeed, the assumption that magnetic surfaces tend also to be equipotential surfaces has been utilized in the design of the potential distribution in many magnetic devices, including the Hall thruster [1], plasma lens [2,3], tandem mirror [4], centrifugal fusion [5-8], plasma centrifuge [9,10,12] or helicon source [11]. This assumption, also called the assumption of equipotentialization of flux surfaces, is thought to hold precisely when the electrons are cold. Finite electron pressure results in a slightly modified potential surface, and under certain conditions a thermalized potential (potential plus a finitetemperature correction) instead is assumed to follow the magnetic surface [1]. The equipotentialization condition implies that radial electric fields capable of rotating plasma can be produced in mirror geometry by specifying the potential along flux surfaces using end electrodes.

The Hall-effect thruster is a particularly well-studied device that exploits the fact that magnetic surfaces tend also to be equipotential surfaces. This device, depicted schematically in figure 1, features largely radial magnetic fields across which an axial potential is applied. The radial magnetic fields are generated in an annular region bounded by magnetic poles. If the magnetic field is large enough to magnetize electrons but not ions, then the electrons $E \times B$ rotate in the azimuthal direction, while ions are accelerated in the axial direction. The advantage of this device over an ion diode, in which ions fall through a potential in the absence of electrons, is that the acceleration is not space-charge limited. Because the electrons rotate azimuthally, they are held more or less at one axial position, canceling out the space charge of the ions. At each axial position the radial magnetic field comprises an equipotential surface, so that, because these surfaces are perpendicular to the axis, the ions are accelerated in the axial direction with little divergence. To the extent that the magnetic surfaces are equipotential surfaces, there is the possibility of controlling the electric potential profile by defining the potential through segmented electrodes at the plasma periphery [13-16]. That the potential 
profile indeed responds measurably to the electrodes supports the picture that the potential tends to equilibrate along field lines.

Considerable effort has been exerted to determine the extent to which the conventional Hall thruster in fact exhibits equipotentialization of the magnetic surfaces, including the deviations due to finite-temperature deviations. Different focusing regimes have been identified [17, 18]. The importance of the magnetic mirroring along magnetic flux surfaces in the Hall thrusters has also been explored $[19,20]$. The mirroring force depends both on the electron pressure as well as the magnetic field gradients. Other methods of narrowing the Hall thruster plume are also based on the equipotentialization of the magnetic surfaces [21]. In each case, in the limit of negligible electron pressure, the force on the ions is thought to be essentially perpendicular to the magnetic surfaces, so that axial ion acceleration is best achieved by purely radial magnetic fields.

What is identified and explained here is the very curious and highly fortuitous effect that, even when the magnetic field diverges considerably, so that the magnetic surfaces are no longer perpendicular at all to the axis, the ion plume has surprisingly little angular divergence, suggesting acceleration that unexpectedly persists largely in the axial direction. We identify two effects whereby cold rotating electrons straighten the thrust vector: first, rotating electrons directly produce a compensating electric field through centrifugal forces. Second, the generation of the Hall magnetic field from rotating electrons similarly necessarily acts to straighten the thrust vector. These two effects are additive.

The paper is organized as follows: In section 2, we call attention to curious observations of remarkably narrow plumes in diverging magnetic fields, a phenomenon which defies any obvious explanation. In section 3 , we review the isorotation theorem for rotating plasma. In section 4 , we show how isorotating electrons produce the helpful and additive deflections of the electric force vector, even in the limit of vanishing electron pressure, in the direction that explains the observations. In section 5, we summarize our main findings.

\section{Surprising results with cylindrical Hall plasma thrusters}

This curious narrowing of the ion acceleration was observed most comprehensively on a Halltype thruster in which the magnetic field lines diverge significantly, a variation known as the cylindrical Hall thruster (CHT) [22]. The CHT differs essentially from conventional annular Hall thrusters in that individual electrons do move considerable distances axially, although, trapped through magneto-electric mirroring, they carry no axial current. The CHT geometry is particularly advantageous when the thruster is miniaturized, since the removal of the central magnetic pole diminishes the surface to volume ratio. Exploiting the expectation that magnetic surfaces are equipotential surfaces, the cusp configuration of the CHT retains radially directed magnetic field surfaces thereby to produce axial electric fields to accelerate ions with minimum plume divergence. Thus, the cusp magnetic configuration shown in figure $2(b)$ was thought to be more promising than the configuration of figure $2(a)$. In fact, the CHT was originally designed to accentuate the cusp configuration [22]. Interesting variations on the cusped thruster configuration have also been explored recently [23].

The CHT in cusp geometry features high ionization efficiency, quiet operation, ion acceleration in a large volume-to-surface ratio channel, and performance comparable to the state-of-the-art conventional Hall thrusters [24]. Recently, by overrunning the discharge current, these thrusters also featured very significant plume narrowing, accompanied by significantly enhanced efficiencies [25]. In this so-called current overrun regime, the cathode supplies extra electrons. These regimes are characterized by increased electric field in the thruster channel, plasma plume narrowing, exceptional efficiencies, and certain differences in 


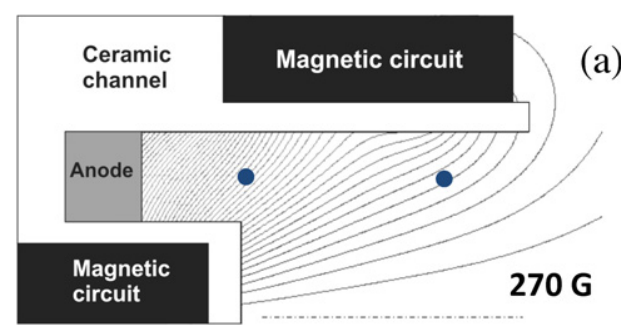

800 G

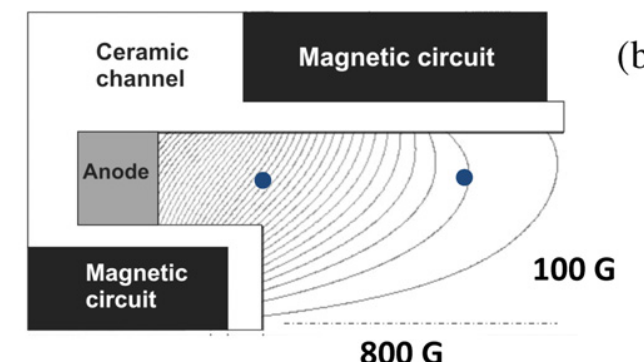

$800 \mathrm{G}$

Figure 2. Magnetic field lines in the CHT. ( a ) Without cusp (direct). (b) With cusp. The lower line in each case is the axis of symmetry. The magnetic field strengths are taken at the position indicated by shaded circles.

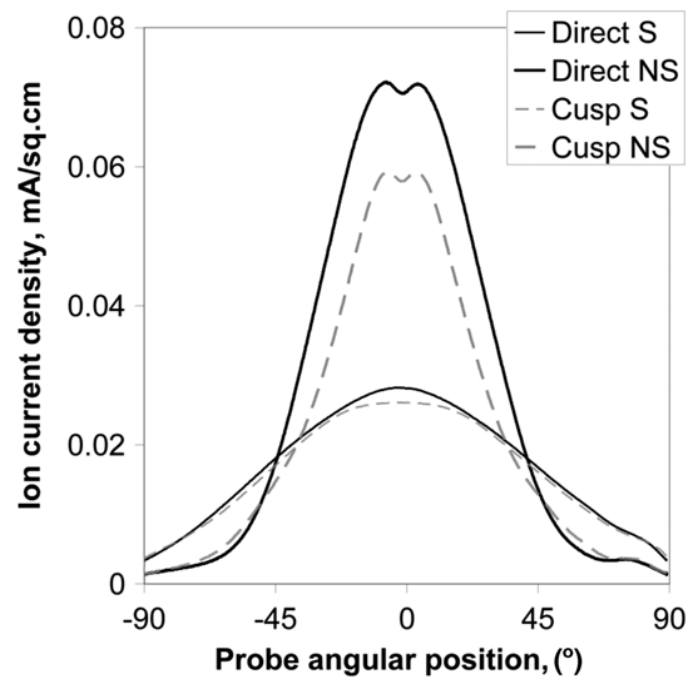

Figure 3. Plume narrowing in the current overrun regime in both the direct and cusp configuration (from [27]). The overrun regime is denoted by $N S$ and the normal regime is denoted by $S$.

temperature and density profiles [26]. As can be seen from figure 3, the plume narrows in the current overrun regime, both for the cusp configuration and the no-cusp or what we call the direct configuration [27].

However, not only was it surprising that the plume consistently narrowed in the current overrun regime, it was also surprising that the plume narrowing was significant both in the cusp and the direct configuration. Even more surprising was that the direct configuration would have about as narrow a plume as the cusp configuration both in the overrun and non-overrun regimes. The surprise was not only that the plumes were similarly narrowed in each configuration in the current overrun regime, but that both enjoyed such narrow plumes even without the current overrun. From figures 2, one should certainly expect very much more divergence if the electric fields were indeed perpendicular to the magnetic surfaces. For thruster applications, the results are certainly welcome, but inexplicable if the equipotential surfaces coincide with the imposed magnetic surfaces.

It thus remains to find not only mechanisms for plume narrowing by insertion of extra electrons at the cathode, but also why the direct and cusp configurations both give such narrow 


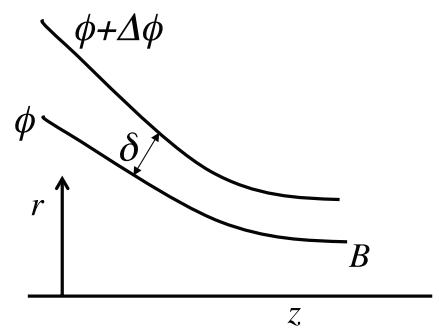

Figure 4. Isorotation theorem. The $z$-axis is the axis of symmetry for the azimuthally symmetric magnetic surfaces.

plumes. Some of the explanation may lie in exactly where the gas becomes ionized, but that cannot be the full explanation. The plume indicates that the force vector on ions is mainly axial, and clearly it should not be if magnetic surfaces are equipotential surfaces.

\section{Preliminaries: isorotation theorem}

In order to lay the groundwork for identifying the mechanisms that deflect the thrust vector so as to make the acceleration more axial, or what we might describe as straightening the thrust vector, it is useful to recall the isorotation theorem. Magnetic surfaces that are also equipotential surfaces have very interesting properties: by the isorotation theorem, any species undergoing $E \times B$ rotation will isorotate on the surface, meaning that the angular frequency of $E \times B$ rotation on each magnetic surface is the same, even at different radii of rotation. Thus, while $E \times B$ rotation velocities differ on the surfaces, the rotation frequencies do not.

If magnetic surfaces are also equipotential surfaces, then the magnetic surfaces also isorotate, by which we mean that the drift velocity divided by the radius is a constant on the surface. To see this, following [5], consider two magnetic surfaces separated by $\delta$ as in figure 4. Since $\boldsymbol{B} \equiv \nabla \times \boldsymbol{A}$, where $\boldsymbol{A}=\hat{\theta} A_{\theta}(r, z)$ we have by Stokes theorem

$$
\oint \boldsymbol{A} \cdot \mathrm{d} s=\int \boldsymbol{B} \cdot \mathrm{d} \boldsymbol{a} .
$$

Taking the contour integrals over the annulus bounded by the magnetic surfaces, at constant $z$, we then have,

$$
2 \pi \Delta\left[r A_{\theta}\right]=2 \pi r B \delta .
$$

In other words, the difference in $r A_{\theta}$ between the two surfaces, $\Delta\left[r A_{\theta}\right]$, is just the enclosed flux $2 \pi r B \delta$. The electric field $E$ between the surfaces is just $\Delta \phi / \delta$, where $\Delta \phi$ is the potential difference. The angular frequency of circulating the magnetic surface at radius $r$ and drift velocity $E / B$ can then be written as

$$
\Omega \equiv E / r B=\Delta \phi / \Delta\left[r A_{\theta}\right]
$$

which is a constant on the flux surfaces since it is the ratio of the differences of quantities that are all constant on the flux surface.

The reasoning that suggests that magnetic surfaces are equipotential surfaces relies upon the notion that electrons can freely move along magnetic surfaces, but not perpendicular to the surface. Therefore, any electric field parallel to the surface would get shorted out by the electron motion. 


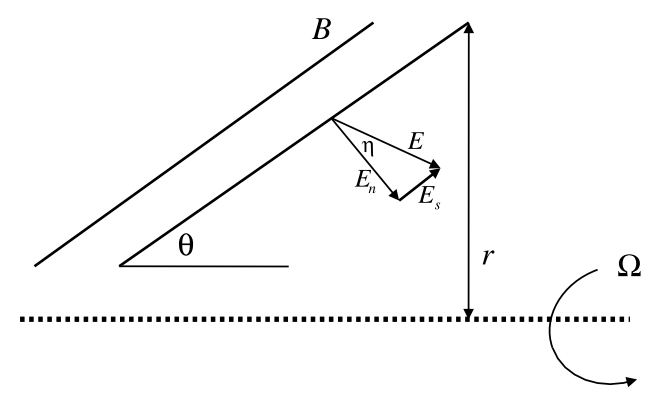

Figure 5. Deflection of force vector $E_{n}$ through angle $\eta$ due to centrifugal forces arising from supersonic rotation with frequency $\Omega$ about axis (dashed line) of rotation.

\section{Deflection of force vector}

In a Hall-type thruster, plasma conditions can affect the ion plume either through the voltage distribution or through the point of introduction of the ions into the force field, for example, through ionization. Here, we will consider the force field. The common assumption that magnetic surfaces are equipotential surfaces leads to a force field that is perpendicular to the magnetic surfaces. While this may be a good assumption for non-rotating magnetized plasma, it may not be a good assumption for rotating plasma. We now show that two effects lead to what we can call a deflection of the force vector from being normal to the magnetic surface, the additive effect of which can be large.

\subsection{Supersonic rotation of electrons}

The first deflection of the thrust vector is due to the centrifugal forces of the rotating electrons. Consider, as in figure 5, that magnetic surfaces are straight, making an angle $\theta$ with the axis of rotation. Assume as a starting point that magnetic surfaces are equipotential surfaces, so that the isorotation theorem holds. Consider electron motion at a point $(r, z)$ near where the electric field is $E_{n}$, which must be normal to the magnetic surface. Since electrons are magnetized, they isorotate with frequency $\Omega=E_{n} / r B$, experiencing therefore a centrifugal radial force $F_{\mathrm{c}}=m r \Omega^{2}$. The force perpendicular to the field line results only in a modified rotation velocity; the force parallel to the field line accelerates electrons radially outward (along the field lines) until that outward force is balanced by an electric field parallel to the surface $E_{\mathrm{s}}$, such that $e E_{\mathrm{s}}=-F_{\mathrm{c}} \sin \theta$. Since the ions are not magnetized, they are not affected by the magnetic field, and hence ions are sensitive to the electric field only. However, as seen from figure 5, the addition of the field $E_{\mathrm{s}}$ results in total field $E$, which represents a deflection of the original electric field $E_{\mathrm{n}}$ through an angle $\eta$. We can thus write the deflection angle as

$$
\eta \simeq \sin \eta=\frac{E_{\mathrm{s}}}{E_{\mathrm{n}}}=\frac{\Omega}{\Omega_{\mathrm{e}}} \sin \theta=\frac{\rho_{\mathrm{L}}}{r} \frac{E / B}{v_{\mathrm{T}}} \sin \theta,
$$

where we defined the gyrofrequency $\Omega_{\mathrm{e}} \equiv e B / m$, and, in the last equality, rewrote the frequency ratio in terms of the Larmor radius $\rho_{\mathrm{L}}=v_{\mathrm{T}} / \Omega_{\mathrm{e}}$, where $v_{\mathrm{T}}$ is the electron thermal speed (in the rotating frame). (We refer to the electron temperature, notwithstanding that the effect is independent of electron temperature, in order to compare the rotation speeds to sonic speeds.) Note that because of the isorotation theorem, the rotation is the same over each magnetic field surface, meaning that on each surface the deflection varies inversely with the strength of the magnetic field. 


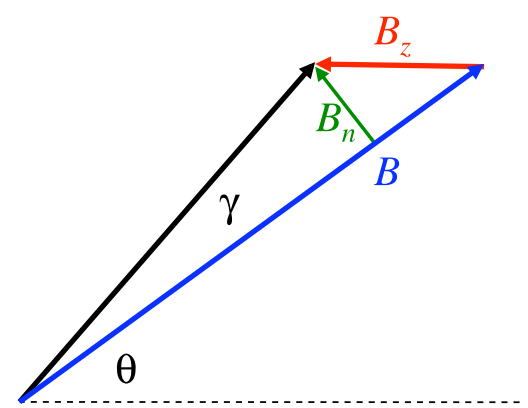

Figure 6. Deflection of thrust vector due to Hall current

To see how large the effect can be, it is convenient to use normalized variables: $T_{\mathrm{e}}=T_{20}(20 \mathrm{eV}) ; B=B_{100}(100 \mathrm{G}) ; E=E_{200}\left(200 \mathrm{~V} \mathrm{~cm}^{-1}\right) ; r=r_{10}(10 \mathrm{~mm})$. Thus, we can write $\rho_{\mathrm{L}} / r=0.1 T_{20}^{1 / 2} / B_{100} r_{10}$ and $E / B v_{\mathrm{T}} \simeq E_{200} / T_{20}^{1 / 2} B_{100}$. For typical numbers for a CHT: $T_{\mathrm{e}}=20 \mathrm{eV} ; B=100 \mathrm{G} ; E=200 \mathrm{~V} \mathrm{~cm}^{-1}$, the rotation is approximately sonic at a location where we might have, say $r=5 \mathrm{~mm}$ and say $\theta=45^{\circ}$. For sonic rotation, we find from equation (4) that $\eta \simeq 8^{\circ}$. What this means is that the parallel electric field that balances sonic rotation of electrons also acts to deflect the thrust vector for ions by about $8^{\circ}$. Were the rotation supersonic, the effect would be correspondingly larger. From the data available, we imagine that the rotation is roughly sonic. Indeed, were it supersonic, the probe measurements made to date, not being directional, would not distinguish the rotation component from the thermal components.

Note that the several degrees are already a significant deflection, even if it does not seem quite large enough to explain fully the fortuitous result, which appears to be somewhat larger. However, it is in the right direction; the numbers are just approximate; and it is not the only new deflection effect to consider. Note also that this calculation is perturbative. We began with the assumption that flux surfaces are equipotential surfaces; thus isorotation could be deduced to hold. But isorotation produces centrifugal forces that in steady state could be only balanced by fields within the flux surfaces. These fields, of course, violate the assumption that the magnetic surfaces are equipotential surfaces, but to the extent that these fields parallel to the magnetic flux surfaces are small, the magnetic flux surfaces will very nearly be equipotential surfaces, which therefore will very nearly obey isorotation.

\subsection{Hall-current deflection of magnetic surface}

The second deflection of the thrust vector is due to the fact that the rotating electron cloud itself produces a self-consistent magnetic field through the Hall current. The generated field then adds to the imposed fields to change the angle of the magnetic surface. To calculate this effect, consider that electrons rotate at speed $E / B$, generating an axial magnetic field, $B_{z}$. For simplicity, assume that the rotation is approximately at constant angular frequency $\Omega$ (solid body rotation), so that we can approximate $B_{z} \simeq e \mu_{0} n \Omega\left(r_{0}^{2}-r^{2}\right) / 2$, where $r_{0}$ is the external radius. Note from figure 6 , that the resultant $z$-directed magnetic field is in the direction so as to make more radial the flux surfaces of the nozzle-like magnetic field characteristic of the CHT. Hence, this field acts in the same direction (straightening the thrust vector) as does the effect of centrifugal forces of the supersonically rotating electrons. 
To estimate this effect, note that for the solid-body rotation of electrons, we have

$$
\frac{B_{z}}{B} \simeq \frac{1}{2}\left(\frac{r_{0}}{\rho_{\mathrm{e}}}\right)^{2} \frac{\Omega}{\Omega_{\mathrm{e}}}\left(1-\frac{r^{2}}{r_{0}^{2}}\right)
$$

where we defined $\rho_{\mathrm{e}} \equiv c / \omega_{\mathrm{pe}}$ as the electron skin depth. From figure 6 , we find the added normal component to the magnetic field, $B_{\mathrm{n}}=B_{z} \sin \theta$, and calculate the deflection angle

$$
\gamma \simeq \frac{B_{\mathrm{n}}}{B}=\frac{B_{z}}{B} \sin \theta=\frac{1}{2}\left(\frac{r_{0}}{\rho_{\mathrm{e}}}\right)^{2}\left(1-\frac{r^{2}}{r_{0}^{2}}\right) \frac{\Omega}{\Omega_{\mathrm{e}}} \sin \theta .
$$

Evidently, by comparing with equation (4), we have $\gamma=0.5 \eta\left(r_{0}^{2}-r^{2}\right) / \rho_{\mathrm{e}}^{2}$. We can write $\rho_{\mathrm{e}} \simeq 5 n_{12}^{-1 / 2} \mathrm{~mm}$, where $n_{12}$ is the density normalized to $10^{12} \mathrm{~cm}^{-3}$. Thus, for densities on the order of $10^{12} \mathrm{~cm}^{-3}$, and say $r_{0} \simeq 10 \mathrm{~mm}$, but $r \simeq 5 \mathrm{~mm}$, the deflection due to the Hall field would be about $150 \%$ of the deflection due to supersonic rotation. So for the example above of sonic rotation and $n_{12}=1$, at $r \simeq 5 \mathrm{~mm}$, we have $\gamma \simeq 12^{\circ}$. This is additional to the $8^{\circ}$ deflection, so the total deflection for these parameters is about $20^{\circ}$. This is already a very significant deviation from the common assumption that the thrust would be normal to the flux surface established by the external magnetic circuit.

\section{Discussion and conclusions}

We identify here two effects in self-organizing rotating plasma that both necessarily tend to make electric forces more axial, thereby explaining hitherto very surprising observations. These effects, both remaining even in the limit of vanishing electron pressure, are additive: first, in equilibrium the supersonic electron rotation must be balanced by an electric field parallel to what to first approximation is the equipotential surface. Second, the supersonic electron rotation generates an axial magnetic field that adds to the applied field magnetic field so as to deflect the magnetic surface. Together these effects add up to considerable deflection of the thrust vector from normal to the magnetic surface, making it more axial, in contrast to the prevailing wisdom.

The effects tend to be comparable at average radii in the example given of the cylindrical Hall thruster, but the effect due to the centrifugal force should dominate at large radii where the centrifugal forces are larger, while the effect due to the Hall magnetic field will dominate at small radii where the largest corrective axial magnetic fields are generated. Even without quantitative calculations, the most quizzical observations of all, namely that the direct and cusped cylindrical Hall thrusters exhibit similar plume divergence, now appear after all not to be so unusual at all.

These effects are all additional to finite-temperature effects, which for sonic rotation would be comparable, but harder to assess, since only for very particular assumptions on the pressure tensor can a thermalized potential be constructed [1]. However, the deflection due to pressure effects in an annular geometry would likely exhibit an inner-outer asymmetry, while centrifugal forces, always pointing outward, give the same deflection direction. Of course, for supersonic rotation, the deflections identified here would dominate. In any event, to decrease the plume divergence, any optimization of the magnetic configuration for Hall thrusters, whether cylindrical or annular, must take into account the effects identified here. Moreover, these new effects may occur for any rotating plasma, and hence may be of importance in a variety of applications. 


\section{Acknowledgments}

This work was supported by AFOSR, by DOE under contract DE-AC02-09CH-11466, and by the US-Israel Binational Science Foundation under Grant No 200822.

\section{References}

[1] Morozov A I and Savelyev V V 2000 Fundamentals of stationary plasma thruster theory Reviews of Plasma Physics vol 21 ed B B Kadomtsev and V D Shafranov (New York: Consultants Bureau)

[2] Morozov A I 2003 Plasma Phys. Rep. 29235

[3] Goncharov A A, Evsyukov A N and Litovko I V 2009 IEEE Trans. Plasma Sci. 371283

[4] Severn G D and Hershkowitz N 1992 Phys. Fluids B 43210

[5] Lehnert B 1971 Nucl. Fusion 11485

[6] Huang Y M and Hassam A B 2001 Phys. Rev. Lett. 87235002

[7] Volosov V I 2006 Nucl. Fusion 46820

[8] Fetterman A J and Fisch N J 2008 Phys. Rev. Lett. 101205003

[9] Krishnan M, Geva M and Hirschfield J L 1981 Phys. Rev. Lett. 4636

[10] Ohkawa T and Miller R 2002 Phys. Plasmas 95116

[11] Lafleur T, Takahashi K, Charles C and Boswell R W 2011 Phys. Plasmas 18080701

[12] Fetterman A J and Fisch N J 2011 Phys. Plasma 18094503

[13] Raitses Y, Dorf L A, Litvak A A and Fisch N J 2000 J. Appl. Phys. 881263

[14] Fisch N J, Raitses Y, Dorf L A and Litvak A A 2001 J. Appl. Phys. 892040

[15] Fruchtman A and Fisch N J 2001 Phys. Plasma 856

[16] Fruchtman A, Fisch N J and Raitses Y 2001 Phys. Plasmas 81048

[17] Hofer R R, Jankovsky R S and Gallimore A D 2006 J. Propul. Power 22721

[18] Linnell J A and Gallimore A D 2006 Phys. Plasmas 13103504

[19] Keidar M and Boyd I D 2005 Appl. Phys. Lett. 87121501

[20] Fossum E C and King L B 2008 IEEE Trans. Plasma Sci. 362088

[21] Fruchtman A and Cohen-Zur A 2006 Appl. Phys. Lett. 89111501

[22] Raitses Y and Fisch N J 2001 Phys. Plasmas 82579

[23] MacDonald N A et al 2011 J. Phys. D: Appl. Phys. 44295203

[24] Smirnov A, Raitses Y and Fisch N J 2007 Phys. Plasma 14057106

[25] Raitses Y, Smirnov A and Fisch N J 2007 Appl. Phys. Lett. 90221502

[26] Smirnov A, Raitses Y and Fisch N J 2008 IEEE Trans. Plasma Sci. 361998

[27] Raitses Y, Smirnov A and Fisch N J 2009 Phys. Plasma 16057106 